\title{
Microwave Consolidation of Photosensitive Planar Glass Layers
}

\author{
P.C. Gow, C. Holmes, Q.S. Ahmed, P.G.R. Smith \\ Optoelectronics Research Centre, University of Southampton, Southampton, SO17 1BJ, UK \\ p.gow@soton.ac.uk
}

\begin{abstract}
Consolidation of photosensitive doped silica glass using a microwave is presented. The work deposits planar glass layers through Flame Hydrolysis Deposition and direct writes Bragg gratings within them using a 244nm laser after consolidation. () 2020 The Author(s)
\end{abstract}

\section{Introduction}

Planar silica is an established platform for fabricating photonic devices as it exhibits low loss and can support waveguide modes which match with optical fibre [1,2]. The planar silica layers are typically fabricated by PECVD or Flame Hydrolysis Deposition (FHD). FHD uses the reaction of $\mathrm{SiCl}_{4}$ in an Oxygen-Hydrogen flame to deposit a silica soot on the surface of a suitable substrate (Fig 1(a)) and is capable of depositing low loss silica films [3]. Dopants such as Ge and B are added to control the photosensitivity and refractive index of the deposited material. The silica soot typically requires consolidation in a furnace at around $1360^{\circ} \mathrm{C}$ for several hours to achieve a fused glass layer. This process can be repeated to form multiple layers of varying photosensitivity, refractive index and thickness. However, these long periods within a high temperature furnace can lead to blending of the deposited silica layers. This results in differences in the target refractive indexes due to diffusion of dopants across layers and can have an affect on the photosensitivity of the glass. Longer cooling times are also associated with crystal growth, which can contribute to increased propagation loss. Consolidation through other means than furnace consolidation, such as with a $\mathrm{CO}_{2}$ laser [4], has completely different thermodynamics, which would likely affct internal stresses, photosensitivity and diffusion.. Here we present a technique using a SiC-lined kiln with low thermal mass within a standard kitchen microwave (Sharp R270SLM) to rapidly heat and cool a silica soot layer, achieving consolidation in under 20 minutes.
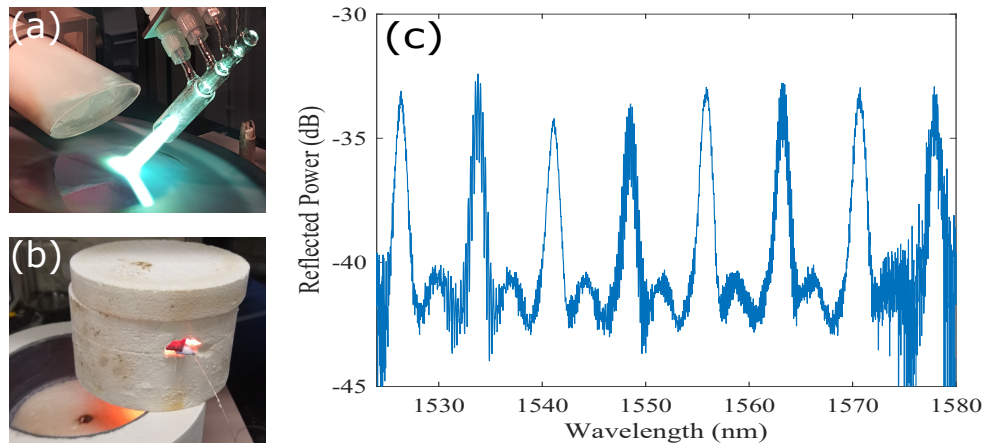

Fig. 1. (a) shows the FHD process applying silica soot to the surface of a silicon wafer. (b) shows the $\mathrm{SiC}$-lined kiln cooling after heating within the microwave. (c) shows an OSA trace of the reflection from the Bragg gratings UV written within the microwave consolidated doped silica.

\section{Method and results}

The substrates used were diced from a silicon wafer with a $15 \mu \mathrm{m}$ thick thermal oxide. A silica layer doped with Germanium and Boron was deposited onto these through FHD. One of the substrates was consolidated by the typical route; within a furnace for several hours at a temperature of $\sim 1360^{\circ} \mathrm{C}$. The second substrate was consolidated using a SiC-lined microwave kiln for 18 minutes before being left to cool for a further 30 minutes. Fig 1(b) shows the kiln cooling after microwave processing. A Metricon prism coupler system was used to measure the thickness and refractive indices of the consolidated layers. An estimate for the propagation losses was also 
made, though this loss includes that of the thermal oxide as well. The results of these measurements are shown in table 1. Microwave consolidation resulted in a thinner layer with higher refractive index. This is likely due to the much faster processing time and higher temperature gradients, which can have a large affect on the resultant fused silica layer [5]. This may also be due to the microwave kiln achieving much higher temperatures than the furnace. In failed attempts the temperature was high enough to melt the silicon substrate $\left(\sim 1414^{\circ} \mathrm{C}\right)$.

The microwave consolidated chip was left in a Hydrogen pressure cell at 120 bar for several days to increase its photosensitivity prior to writing [6]. It was then direct UV written with a CW frequency-doubled argon ion laser operating at $244 \mathrm{~nm}$ using the small-spot technique [7]. This enabled the simultaneous definition of single-mode waveguides and Bragg gratings within the photosensitive silica. 11 waveguides were written with an average laser power of $30 \mathrm{~mW}$ and fluences ranging from 0.5 to $21 \mathrm{~kJ} \mathrm{~cm}^{-2}$. Each waveguide contained eight $1.5 \mathrm{~mm}$ long Bragg gratings with wavelengths between 1520 and $1580 \mathrm{~nm}$. The chip was then annealed overnight at $90{ }^{\circ} \mathrm{C}$ for 16 hours to allow the remaining Hydrogen to outgas. Broadband light from an erbium-doped fiber ASE source was polarized and coupled into the waveguides via a PM fiber V-groove assembly. The back reflected light from the gratings was measured with an OSA and the trace taken from the waveguide written with a fluence of $13 \mathrm{~kJ}$ $\mathrm{cm}^{-2}$ is shown in fig 1 (c). The peak reflectivity of these weak gratings was measured as $0.05 \%$. Both TE an TM polarization was coupled into the waveguides and the average shift in peak wavelength of the gratings was $\sim 0.5$ $\mathrm{nm}$, giving a birefringence of $9.03 \times 10^{-4}$. The Bragg gratings were used for measuring propagation loss [8]. The losses measured from the waveguides gave a best value of $0.81 \pm 0.35 \mathrm{~dB} \mathrm{~cm}^{-1}$. This is lower than the Metricon measurements, which is due to the fact this measures the loss of a single $10 \mu \mathrm{m}$ width waveguide defined within the guiding layer, whereas the Metricon measures the combined loss of the film and thermal oxide. The waveguides are also exposed to the air, so a lower loss would be achieved with deposition of a cladding layer on top.

Table 1. Process information and Metricon prism coupler results from the furnace and microwave consolidated chips. Errors shown are the standard deviation, which is negligible for refractive index.

\begin{tabular}{ccccc}
\hline Consolidation & Process Time & Thickness $(\mu \mathrm{m})$ & Index & Loss $\left(\mathrm{dB} \mathrm{cm}^{-1}\right)$ \\
\hline Furnace & $\sim 8$ Hours & $3.804 \pm 0.017$ & 1.446 & $2.28 \pm 0.28$ \\
Microwave & 18 Minutes $(+30$ minutes cooling) & $3.386 \pm 0.085$ & 1.447 & $2.41 \pm 0.32$ \\
\hline
\end{tabular}

\section{Conclusion}

A silica soot layer was fused in a $\mathrm{SiC}$ microwave kiln. Consolidation and cooling took a total of 48 minutes, reducing consolidation time by several hours hours compared to furnace processing. The resulting planar silica layer is capable of hosting Waveguides and Bragg gratings fabricated through UV writing. We shall present differences founded between furnace and microwave consolidated FHD in terms of layer diffusion, birefringence, photosensitivity, density and loss.

\section{References}

1. M. R. Poulsen, P. I. Borel, J. Fage-Pedersen, J. Huebner, M. Kristensen, J. H. Povlsen, K. Rottwitt, M. Svalgaard, and W. Svendsen, "Advances in silica-based integrated optics," Opt. Eng. 42, 2821-2835 (2003).

2. A. Himeno, K. Kato, and T. Miya, "Silica-based planar lightwave circuits," IEEE J. selected topics quantum electronics 4, 913-924 (1998).

3. J. M. Ruano, V. Benoit, J. S. Aitchison, and J. M. Cooper, "Flame hydrolysis deposition of glass on silicon for the integration of optical and microfluidic devices," Anal. chemistry 72, 1093-1097 (2000).

4. P. C. Gow, A. Jantzen, K. Boyd, N. Simakov, J. Daniel, A. C. Gray, J. C. Gates, P. Shardlow, P. G. Smith, and C. Holmes, "Consolidation of flame hydrolysis deposited silica with a $9.3 \mu \mathrm{m}$ wavelength co2 laser," Electron. Lett. 54, 945-947 (2018).

5. G. Barbarossa and P. J. Laybourn, "Effect of temperature gradient on sintering kinetics of doped silica waveguides by flame hydrolysis deposition," in Integrated Optical Circuits II, , vol. 1794 (International Society for Optics and Photonics, 1993), pp. 191-197.

6. M. Fokine and W. Margulis, "Large increase in photosensitivity through massive hydroxyl formation," Opt. letters 25, 302-304 (2000).

7. C. Sima, J. C. Gates, H. L. Rogers, P. L. Mennea, C. Holmes, M. N. Zervas, and P. G. R. Smith, "Ultra-wide detuning planar Bragg grating fabrication technique based on direct UV grating writing with electro-optic phase modulation," Opt. express 21, 15747-15754 (2013).

8. H. L. Rogers, S. Ambran, C. Holmes, P. G. R. Smith, and J. C. Gates, "In situ loss measurement of direct UV-written waveguides using integrated Bragg gratings,” Opt. letters 35, 2849-2851 (2010). 